Louisiana State University

LSU Digital Commons

$12-1-2004$

\title{
Characterization of probability measures through the canonically associated interacting fock spaces
}

\author{
Luigi Accardi \\ Università degli Studi di Roma Tor Vergata \\ Hui Hsiung Kuo \\ Louisiana State University \\ Aurel Stan \\ University of Rochester
}

Follow this and additional works at: https://digitalcommons.Isu.edu/mathematics_pubs

\section{Recommended Citation}

Accardi, L., Kuo, H., \& Stan, A. (2004). Characterization of probability measures through the canonically associated interacting fock spaces. Infinite Dimensional Analysis, Quantum Probability and Related Topics, 7(4), 485-505. https://doi.org/10.1142/S0219025704001736

This Article is brought to you for free and open access by the Department of Mathematics at LSU Digital Commons. It has been accepted for inclusion in Faculty Publications by an authorized administrator of LSU Digital Commons. For more information, please contact ir@lsu.edu. 
Infinite Dimensional Analysis, Quantum Probability

and Related Topics

Vol. 7, No. 4 (2004) 485-505

(C) World Scientific Publishing Company

\title{
CHARACTERIZATION OF PROBABILITY MEASURES THROUGH THE CANONICALLY ASSOCIATED INTERACTING FOCK SPACES
}

\author{
LUIGI ACCARDI \\ Centro Vito Volterra, Università di Roma "Tor Vergata", \\ 00133 Roma, Italy \\ accardi@volterra.mat.uniroma2.it \\ HUI-HSIUNG KUO \\ Department of Mathematics, Louisiana State University, \\ Baton Rouge, LA 70803, USA \\ kuo@math.lsu.edu \\ AUREL STAN \\ Department of Mathematics, University of Rochester, \\ Rochester, NY 14627, USA \\ astan@math.rochester.edu \\ Received 11 June 2003 \\ Revised 2 July 2003 \\ Communicated by T. Hida
}

\begin{abstract}
We continue our program of coding the whole information of a probability measure into a set of commutation relations canonically associated to it by presenting some characterization theorems for the symmetry and factorizability of a probability measure on $\mathbb{R}^{d}$ in terms of the canonically associated interacting creation, annihilation and number operators.
\end{abstract}

Keywords: Probability measure; orthogonal polynomials; polynomially symmetric; polynomially factorizable.

AMS Subject Classifications: 42C05, 46L53

\section{Introduction}

The notion of interacting Fock space (IFS) was introduced in Ref. 2 and axiomatized in Ref. 3 where it was conjectured that the category of IFS could play for general probability measures the same role as the usual Fock space for the Gaussian measures. The first confirmation of this conjecture came from the paper ${ }^{1}$ of Accardi and Bożejko who showed that the theory of one-mode interacting Fock spaces is 
canonically isomorphic to the theory of orthogonal polynomials in one variable. The isomorphism is canonical in the sense that it carries the multiplication operator by the independent variable to a linear combination of creation, annihilation, and number operators on the corresponding interacting Fock space.

The problem to extend the Accardi-Bożejko isomorphism to the case of several variables has been recently solved by Accardi and $\mathrm{Nahni}^{4}$ and extended to the infinite dimensional case by Accardi, Kuo and Stan. A new feature in the multi-mode case is that not all interacting Fock spaces are canonically isomorphic to spaces of orthogonal polynomials. Those being so are characterized in terms of a sequence of quadratic commutation relations among finite dimensional matrices. Moreover, the quantum decomposition of an arbitrary vector-valued random variable with finite moments of any order can be easily written down as a sum of creation, annihilation and number operators.

This result opens the way to the program of coding the whole information of a probability measure into a set of commutation relations canonically associated to it in full analogy to what happens with the codification of the properties of a Gaussian measure into the Heisenberg commutation relations plus the Fock property.

In this paper we begin to realize this program by showing how some properties of a probability measure on $\mathbb{R}^{d}$ are reflected by the actions of its creation, annihilation, and number operators.

\section{Fundamental Identities}

Let $d \in \mathbb{N}$ be fixed. Let $\mu$ be a probability measure on the Borel subsets of $\mathbb{R}^{d}$. Throughout this paper we fix the canonical basis in $\mathbb{R}^{d}$ and identify vectors $x=$ $\left(x_{1}, \ldots, x_{d}\right) \in \mathbb{R}^{d}$ with ordered $d$-tuples of real numbers. However, all the results below can be formulated in an intrinsic, i.e. coordinate independent way and this will be discussed in a future paper. We assume that $\mu$ has finite moments of all orders, i.e. for all $1 \leq p<\infty$ and $j \in\{1,2, \ldots, d\}, \int_{\mathbb{R}^{d}}\left|x_{j}\right|^{p} \mu(d x)<\infty$. We denote the inner product on $L^{2}\left(\mathbb{R}^{d}, \mu\right)$ by $\langle$,$\rangle .$

Let $F_{0}=\mathbb{C} \cdot 1$ be the complex multiples of the constant function equal to 1 in $L^{2}\left(\mathbb{R}^{d}, \mu\right)$, and for $n \geq 1$ let $F_{n}$ be the complex vector space of all polynomial functions of variables $x_{1}, x_{2}, \ldots, x_{d}$ of degree less than or equal to $n$. We have:

$$
F_{0} \subset F_{1} \subset F_{2} \subset \cdots \subset F_{n} \subset \cdots \subset L^{2}\left(\mathbb{R}^{d}, \mu\right) .
$$

Let $G_{0}=\mathbb{C} \cdot 1$ and for $n \geq 1$ let $G_{n}$ be the orthogonal complement of $F_{n-1}$ in $F_{n}$. Notice that $F_{n-1}$ and $F_{n}$ are finite dimensional (therefore closed) subspaces of $L^{2}\left(\mathbb{R}^{d}, \mu\right)$. Then the Hilbert spaces $G_{n}, n \geq 0$, are orthogonal subspaces of $L^{2}\left(\mathbb{R}^{d}, \mu\right)$. Let $\mathcal{H}$ denote the orthogonal direct sum of $G_{n}, n \geq 0$ :

$$
\mathcal{H}=\bigoplus_{n \geq 0} G_{n} \quad \text { (Hilbert space sense). }
$$

For any $j \in\{1,2, \ldots, d\}$, we denote by $X_{j}$ the multiplication by $x_{j}$ operator. This operator is densely defined on $\mathcal{H}$. Its domain contains $F_{n}, \forall n \geq 0$, since $\mu$ has finite 
moments of any order. Note that, for every $n \geq 0, X_{j}$ maps $F_{n}$ into $F_{n+1}$ and is a symmetric operator.

Lemma 2.1. For any $j \in\{1,2, \ldots, d\}$ and $n \geq 0$, we have

$$
X_{j} G_{n} \perp G_{k}, \quad \forall k \neq n-1, n, n+1 .
$$

Proof. Let $\phi \in G_{n}$. Then $X_{j} \phi \in F_{n+1}$. Hence $X_{j} \phi \perp G_{k}$ for all $k \geq n+2$. On the other hand, for any $\psi \in G_{k}$ with $k \leq n-2$, we have $X_{j} \psi \in F_{n-1}$ hence, by the symmetry of $X_{j}$

$$
\left\langle X_{j} \phi, \psi\right\rangle=\left\langle\phi, X_{j} \psi\right\rangle=0 .
$$

Thus $X_{j} \phi \perp G_{k}$ for all $k \leq n-2$.

For any $n \geq 0$, let $P_{n}$ denote the orthogonal projection of $\mathcal{H}$ onto $G_{n}$.

Theorem 2.1. (Recurrence relations) For any $j \in\{1,2, \ldots, d\}$ and $n \geq 0$, the following equality holds:

$$
X_{j} P_{n}=P_{n+1} X_{j} P_{n}+P_{n} X_{j} P_{n}+P_{n-1} X_{j} P_{n},
$$

where $P_{-1}=0$ by convention.

Proof. Equation (2.2) is equivalent to

$$
X_{j} \phi=P_{n+1} X_{j} \phi+P_{n} X_{j} \phi+P_{n-1} X_{j} \phi, \quad \forall \phi \in G_{n} .
$$

Let $\phi \in G_{n}$. By Lemma 2.1, $X_{j} \phi$ can be written as

$$
X_{j} \phi=u+v+w,
$$

where $u \in G_{n+1}, v \in G_{n}$ and $w \in G_{n-1}$. Apply $P_{n+1}$ to both sides of Eq. (2.4). Since $P_{n+1} u=u$ and $P_{n+1} v=P_{n+1} w=0$, we get $u=P_{n+1} X_{j} \phi$. Similarly, we can apply $P_{n}$ and $P_{n-1}$ to both sides of Eq. (2.4) to get $v=P_{n} X_{j} \phi$ and $w=P_{n-1} X_{j} \phi$. Thus Eq. (2.3) is proved.

Now for each $j \in\{1,2, \ldots, d\}$ and $n \geq 0$ we define the following operators:

$$
\begin{aligned}
& D_{n}^{+}(j)=P_{n+1} X_{j} P_{n}: G_{n} \rightarrow G_{n+1}, \\
& D_{n}^{0}(j)=P_{n} X_{j} P_{n}: G_{n} \rightarrow G_{n}, \\
& D_{n}^{-}(j)=P_{n-1} X_{j} P_{n}: G_{n} \rightarrow G_{n-1} .
\end{aligned}
$$

We define $F_{-1}$ and $G_{-1}$ to be the null space $\{0\}$.

Theorem 2.2. For any $i, j \in\{1,2, \ldots, d\}$ and $n \geq 0$ the following identities hold:

- $D_{n+1}^{+}(i) D_{n}^{+}(j)=D_{n+1}^{+}(j) D_{n}^{+}(i)$,

- $D_{n+1}^{0}(i) D_{n}^{+}(j)+D_{n}^{+}(i) D_{n}^{0}(j)$

$$
=D_{n+1}^{0}(j) D_{n}^{+}(i)+D_{n}^{+}(j) D_{n}^{0}(i),
$$




$$
\begin{aligned}
& \text { - } D_{n+1}^{-}(i) D_{n}^{+}(j)+D_{n}^{0}(i) D_{n}^{0}(j)+D_{n-1}^{+}(i) D_{n}^{-}(j) \\
& \quad=D_{n+1}^{-}(j) D_{n}^{+}(i)+D_{n}^{0}(j) D_{n}^{0}(i)+D_{n-1}^{+}(j) D_{n}^{-}(i) .
\end{aligned}
$$

Proof. Apply $P_{n+2} X_{i}$ to both sides of Eq. (2.2) to obtain

$$
\begin{aligned}
P_{n+2} X_{i} X_{j} P_{n}= & P_{n+2} X_{i} P_{n+1} X_{j} P_{n}+P_{n+2} X_{i} P_{n} X_{j} P_{n} \\
& +P_{n+2} X_{i} P_{n-1} X_{j} P_{n} .
\end{aligned}
$$

Observe that

$$
\begin{aligned}
P_{n+2} X_{i} P_{n+1} X_{j} P_{n} & =D_{n+1}^{+}(i) D_{n}^{+}(j), \\
P_{n+2} X_{i} P_{n} X_{j} P_{n} & =P_{n+2} X_{i} P_{n-1} X_{j} P_{n}=0 .
\end{aligned}
$$

Therefore, we obtain the equality:

$$
P_{n+2} X_{i} X_{j} P_{n}=D_{n+1}^{+}(i) D_{n}^{+}(j) .
$$

Interchange the role of $i$ and $j$ to get

$$
P_{n+2} X_{j} X_{i} P_{n}=D_{n+1}^{+}(j) D_{n}^{+}(i) .
$$

Since $P_{n+2} X_{i} X_{j} P_{n}=P_{n+2} X_{j} X_{i} P_{n}$, Eqs. (2.11) and (2.12) yield the identity in Eq. (2.8).

Similarly, apply $P_{n+1} X_{i}$ to both sides of Eq. (2.2) to get

$$
\begin{aligned}
P_{n+1} X_{i} X_{j} P_{n}= & P_{n+1} X_{i} P_{n+1} X_{j} P_{n}+P_{n+1} X_{i} P_{n} X_{j} P_{n} \\
& +P_{n+1} X_{i} P_{n-1} X_{j} P_{n} .
\end{aligned}
$$

Observe that

$$
\begin{aligned}
P_{n+1} X_{i} P_{n+1} X_{j} P_{n} & =D_{n+1}^{0}(i) D_{n}^{+}(j), \\
P_{n+1} X_{i} P_{n} X_{j} P_{n} & =D_{n}^{+}(i) D_{n}^{0}(j) \\
P_{n+1} X_{i} P_{n-1} X_{j} P_{n} & =0 .
\end{aligned}
$$

Therefore, we obtain the equality:

$$
P_{n+1} X_{i} X_{j} P_{n}=D_{n+1}^{0}(i) D_{n}^{+}(j)+D_{n}^{+}(i) D_{n}^{0}(j) .
$$

Interchange the role of $i$ and $j$. Since $P_{n+1} X_{i} X_{j} P_{n}=P_{n+1} X_{j} X_{i} P_{n}$, we obtain the identity in Eq. (2.9).

Finally, apply $P_{n} X_{i}$ to both sides of Eq. (2.2) and interchange the role of $i$ and $j$ to obtain the identity in Eq. (2.10).

Proposition 2.1. For any $j \in\{1,2, \ldots, d\}$ and $n \geq 0$ the operators $D_{n}^{+}(j), D_{n}^{0}(j)$ and $D_{n}^{-}(j)$ satisfy the identities:

$$
\left(D_{n}^{+}(j)\right)^{*}=D_{n+1}^{-}(j), \quad\left(D_{n}^{0}(j)\right)^{*}=D_{n}^{0}(j) .
$$


Proof. Since $X_{j}^{*}=X_{j}$ we have the equalities

$$
\begin{aligned}
& \left(D_{n}^{+}(j)\right)^{*}=\left(P_{n+1} X_{j} P_{n}\right)^{*}=P_{n} X_{j} P_{n+1}=D_{n+1}^{-}(j), \\
& \left(D_{n}^{0}(j)\right)^{*}=\left(P_{n} X_{j} P_{n}\right)^{*}=P_{n} X_{j} P_{n}=D_{n}^{0}(j),
\end{aligned}
$$

which prove the proposition.

For each $j \in\{1,2, \ldots, d\}$, we define the densely defined linear operators $a^{+}(j)$, $a^{0}(j)$ and $a^{-}(j)$, on $\mathcal{H}$, by

$$
\left.a^{+}(j)\right|_{G_{n}}=D_{n}^{+}(j),\left.\quad a^{0}(j)\right|_{G_{n}}=D_{n}^{0}(j),\left.\quad a^{-}(j)\right|_{G_{n}}=D_{n}^{-}(j),
$$

for all $n \geq 0$.

Equation (2.2) becomes now:

$$
X_{j}=a^{+}(j)+a^{0}(j)+a^{-}(j), \quad \forall j \in\{1,2, \ldots, d\} .
$$

Theorem 2.3. If $\mu$ is any probability measure on $\mathbb{R}^{d}$ having finite moments of any orders, then $\forall i, j \in\{1,2, \ldots, d\}$

$$
a^{-}(i) a^{-}(j)=a^{-}(j) a^{-}(i), \quad a^{+}(i) a^{+}(j)=a^{+}(j) a^{+}(i) .
$$

Proof. The equality $a^{+}(i) a^{+}(j)=a^{+}(j) a^{+}(i)$ is a restatement of Eq. (2.8). Taking the adjoint on both sides of this relation we obtain $a^{-}(j) a^{-}(i)=a^{-}(i) a^{-}(j)$.

For all $j \in\{1,2, \ldots, d\}$ and $n \geq 1$, we have $a^{-}(j): G_{n} \rightarrow G_{n-1}$. The constant polynomial 1 is called the vacuum vector. We have $a^{-}(j) 1=0, \forall j \in\{1,2, \ldots, d\}$.

Lemma 2.2. Let $j \in\{1,2, \ldots, d\}$. We have $a^{0}(j) 1=0$ if and only if

$$
\left\langle x_{j}\right\rangle:=\int_{\mathbb{R}^{d}} x_{j} \mu(d x)=0 .
$$

Proof. We have

$$
D_{0}^{0}(j) 1=P_{0} X_{j} P_{0} 1=\left\langle x_{j}, 1\right\rangle 1=\left(\int_{\mathbb{R}^{d}} x_{j} \mu(d x)\right) 1 .
$$

Thus $a^{0}(j) 1=0$ if and only if $\int_{\mathbb{R}^{d}} x_{j} \mu(d x)=0$.

\section{Polynomially Symmetric Measures}

For any monomial $x_{1}^{i_{1}} x_{2}^{i_{2}} \cdots x_{d}^{i_{d}}$, we define its degree to be $i_{1}+i_{2}+\cdots+i_{d}$.

Definition 3.1. A measure $\mu$ on $\mathbb{R}^{d}$ is called polynomially symmetric if all of its mixed moments of odd order vanish, i.e. for all monomials $x_{1}^{i_{1}} x_{2}^{i_{2}} \cdots x_{d}^{i_{d}}$ of odd degree, we have $\int_{\mathbb{R}^{d}} x_{1}^{i_{1}} x_{2}^{i_{2}} \cdots x_{d}^{i_{d}} \mu(d x)=0$.

Definition 3.2. A measure $\mu$ on $\mathbb{R}^{d}$ is called symmetric if for any Borel subset $A$ of $\mathbb{R}^{d}$ we have $\mu(A)=\mu(-A)$, where $-A:=\{-x \mid x \in A\}$. 
Observe that if $\mu$ is symmetric, then $\mu$ is also polynomially symmetric. The converse is not true. In Example 5.1 of Sec. 5 we present a polynomially symmetric measure that is not symmetric.

Let $\mathcal{P}$ be the set of all polynomials in the variables $x_{1}, x_{2}, \ldots, x_{d}$. Let $W_{\text {even }}$ be the vector subspace of $\mathcal{P}$ spanned by the set of all monomials of even degree and $W_{\text {odd }}$ the vector subspace of $\mathcal{P}$ spanned by the set of all monomials of odd degrees.

Let us assume that $\mu$ is polynomially symmetric. If $f \in W_{\text {even }}$ and $g \in W_{\text {odd }}$, then, since $\mu$ is polynomially symmetric, $E[f \bar{g}]=0$, where $E$ denotes the expectation with respect to $\mu$, i.e. the polynomials $f$ and $g$ are orthogonal. Thus $W_{\text {even }} \perp W_{\text {odd }}$. We may apply the Grahm-Schmidt orthogonalization procedure first to $W_{\text {even }}$ and obtain a complete orthonormal set $S_{1}$ for $W_{\text {even }}$. After this we may apply the Grahm-Schmidt orthogonalization procedure to $W_{\text {odd }}$ and obtain a complete orthonormal set $S_{2}$ for $W_{\text {odd }}$. Since $S_{1} \subset W_{\text {even }}, S_{2} \subset W_{\text {odd }}$, and $W_{\text {even }} \perp W_{\text {odd }}$, we have $S_{1} \perp S_{2}$. Thus $S:=S_{1} \cup S_{2}$ is a complete orthonormal set for the space $\mathcal{P}$ of all polynomial functions. Using this complete orthonormal set $S$ for $\mathcal{P}$, we can see that all polynomials in $G_{n}$, when $n$ is even, are linear combinations of monomials of even degree. When $n$ is odd, all polynomials in $G_{n}$ are linear combinations of monomials of odd degree.

Theorem 3.1. If $\mu$ is a probability measure on $\mathbb{R}^{d}$, having finite moments of all orders, then $\mu$ is polynomially symmetric if and only if for all $j \in\{1,2, \ldots, d\}$,

$$
X_{j}=a^{+}(j)+a^{-}(j) .
$$

This means that for all $j \in\{1,2, \ldots, d\}, a^{0}(j)=0$.

Proof. $(\Rightarrow)$ Let us assume that $\mu$ is polynomially symmetric. Let $j \in\{1,2, \ldots, d\}$. To show that $a^{0}(j)=0$, we must prove that for any $n \geq 0, P_{n} X_{j} P_{n}=0$. To prove this, we will show that for any polynomials $f$ and $g$, we have $\left\langle P_{n} X_{j} P_{n} f, g\right\rangle=0$.

Let $n \geq 0$ be fixed. Let $f$ and $g$ be two polynomials. Since $P_{n} f \in G_{n}$ and $P_{n} g \in G_{n}, P_{n} f$ and $P_{n} g$ are linear combinations of monomials that are either all of even degree if $n$ is even or all of odd degree if $n$ is odd. We have:

$$
\begin{aligned}
\left\langle P_{n} X_{j} P_{n} f, g\right\rangle & =\left\langle X_{j} P_{n} f, P_{n} g\right\rangle \\
& =E\left[X_{j}\left(P_{n} f\right) \overline{P_{n} g}\right] \\
& =E\left[\left(x_{j} \sum a_{k_{1} \cdots k_{d}} x_{1}^{k_{1}} \cdots x_{d}^{k_{d}}\right)\left(\sum \overline{b_{l_{1} \cdots l_{d}}} x_{1}^{l_{1}} \cdots x_{d}^{l_{d}}\right)\right] \\
& =E\left[\sum a_{k_{1} \cdots k_{d}} \overline{b_{l_{1} \cdots l_{d}}} x_{1}^{k_{1}+l_{1}} \cdots x_{j}^{k_{j}+l_{j}+1} \cdots x_{d}^{k_{d}+l_{d}}\right] \\
& =\sum a_{k_{1} \cdots k_{d}} \overline{b_{l_{1} \cdots l_{d}}} E\left[x_{1}^{k_{1}+l_{1}} \cdots x_{j}^{k_{j}+l_{j}+1} \cdots x_{d}^{k_{d}+l_{d}}\right] .
\end{aligned}
$$

Observe that:

$$
\begin{gathered}
\left(k_{1}+l_{1}\right)+\cdots+\left(k_{j}+l_{j}+1\right)+\cdots+\left(k_{d}+l_{d}\right) \\
=\left(k_{1}+\cdots+k_{d}\right)+\left(l_{1}+\cdots+l_{d}\right)+1
\end{gathered}
$$




$$
\begin{aligned}
& \equiv n(\bmod 2)+n(\bmod \\
& \equiv(2 n+1)(\bmod 2) \\
& \equiv 1(\bmod 2) .
\end{aligned}
$$$$
2)+1(\bmod
$$

Since $\mu$ is polynomially symmetric, we have:

$$
E\left[x_{1}^{k_{1}+l_{1}} \cdots x_{j}^{k_{j}+l_{j}+1} \cdots x_{d}^{k_{d}+l_{d}}\right]=0 .
$$

$(\Leftarrow)$ Let $\mu$ be a probability measure on $\mathbb{R}^{d}$ having finite moments of all orders, such that for all $1 \leq j \leq d, a^{0}(j)=0$. We will prove by induction on $k$ that for all monomials $m(x)=x_{1}^{i_{1}} x_{2}^{i_{2}} \cdots x_{d}^{i_{d}}$, of degree $2 k+1$, we have $\int_{\mathbb{R}^{d}} m(x) \mu(d x)=0$.

For $k=0$, the only monomials having degree 1 are $m_{j}(x)=x_{j}$, for $1 \leq j \leq d$. Since $a^{0}(j) 1=0$, it follows that $P_{0} X_{j} 1=0$. Since $P_{0}$ is the projection on the onedimensional vector space $\mathbb{C}$ for which 1 is an orthonormal basis, we have $P_{0} X_{j} 1=$ $\left\langle x_{j}, 1\right\rangle 1$. Thus $\left\langle x_{j}, 1\right\rangle=0$, which means $\int_{\mathbb{R}^{d}} x_{j} \mu(d x)=0$, for all $1 \leq j \leq d$.

Let us assume now that the expectation of all monomials of odd degree less than or equal to $2 k-1$ is zero, where $k \geq 1$. We want to prove that the expectation of all monomials of degree $2 k+1$ is 0 . Let $m(x)=x_{1}^{i_{1}} x_{2}^{i_{2}} \cdots x_{d}^{i_{d}}$ be a monomial such that $i_{1}+i_{2}+\cdots+i_{d}=2 k+1$. Let us a choose a number $j \in\{1,2, \ldots, d\}$ such that $i_{j}>0$. We can write $m(x)=x_{j} q(x) r(x)$, where $q(x)$ and $r(x)$ are monomials of degree equal to $k$. Let $F_{k}^{\mathrm{e}}$ be the vector space spanned by the monomials of even degree less than or equal to $k$. Let $F_{k}^{o}$ be the vector space spanned by the monomials of odd degree less than or equal to $k$. According to our induction hypothesis $F_{k}^{\mathrm{e}}$ and $F_{k}^{\mathrm{o}}$ are orthogonal subspaces of $L^{2}\left(\mathbb{R}^{d}, \mu\right)$. Thus if we choose an orthonormal basis $\left\{e_{i}\right\}_{i \in I}$ of $F_{k}^{\mathrm{e}}$ and an orthonormal basis $\left\{f_{j}\right\}_{j \in J}$ of $F_{k}^{\mathrm{o}}$, then $\left\{e_{i}\right\}_{i \in I} \cup\left\{f_{j}\right\}_{j \in J}$ is an orthonormal basis of the space $F_{k}$ of all polynomials of degree less than or equal to $k$. Using this particular basis for $F_{k}$, according to our induction hypothesis, we can see that: $P_{k} q(x)=q(x)-u_{k-2}(x)-u_{k-4}(x)-\cdots$ and $P_{k} r(x)=r(x)-v_{k-2}(x)-$ $v_{k-4}(x)-\cdots$, where $u_{i}$ and $v_{i}$ are linear combinations of monomials of the same degree $i$.

Since $a^{0}(j)=0$, we have $P_{k} X_{j} P_{k} q(x)=0$. Thus we obtain:

$$
\begin{aligned}
0 & =\left\langle P_{k} X_{j} P_{k} q(x), r(x)\right\rangle \\
& =\left\langle X_{j} P_{k} q(x), P_{k} r(x)\right\rangle \\
& =\left\langle x_{j}\left(q(x)-u_{k-2}(x)-u_{k-4}(x)-\cdots\right), r(x)-v_{k-2}(x)-v_{k-4}(x)-\cdots\right\rangle \\
& =\left\langle x_{j} q(x), r(x)\right\rangle-\sum E[w(x)],
\end{aligned}
$$

where all the $w(x)$ are monomials of odd degree less than or equal to $2 k-1$. By the induction hypothesis $E[w(x)]=0$. From the last equality we obtain $\left\langle x_{j} q(x), r(x)\right\rangle=$ 0 . This means $E[m(x)]=0$.

Thus we have proved by induction that the expectation of all monomials of odd degree is zero. Hence $\mu$ is polynomially symmetric. 
Definition 3.3. Let $\mu$ be a probability measure on $\mathbb{R}^{d}$ and $c \in \mathbb{R}^{d}$. We say that $\mu$ is polynomially symmetric about $c$, if for any monomial $m(x)$ of odd degree, we have:

$$
\int_{\mathbb{R}^{d}} m(x-c) \mu(d x)=0 .
$$

Let us observe that if $\mu$ is a polynomially symmetric probability measure on $\mathbb{R}^{d}$, then for any $c=\left(c_{1}, c_{2}, \ldots, c_{d}\right) \in \mathbb{R}^{d}$, the probability measure $\mu_{c}$, defined by $\mu_{c}(B):=\mu(B-c)$, where $B$ is a Borel subset of $\mathbb{R}^{d}$ and $B-c:=\{x-c \mid x \in B\}$, is polynomially symmetric about $c$. We do the same construction for $\mu_{c}$ as we did for $\mu$, and call the corresponding spaces $\tilde{G}_{n}, n \geq 0$, and the corresponding operators $\tilde{P}_{n}, n \geq 0, \tilde{a}^{-}, \tilde{a}^{0}$, and $\tilde{a}^{+}$. Since for any integrable function $f$ with respect to $\mu$, we have $\int_{\mathbb{R}^{d}} f(x) \mu(d x)=\int_{\mathbb{R}^{d}} f(x-c) \mu_{c}(d x)$, we can see that a polynomial $Q(x)$ belongs to $G_{n}$ if and only if $Q(x-c)$ belongs to $\tilde{G}_{n}$. Moreover, $\left\{e_{i}(x)\right\}_{i \in I}$ is an orthonormal basis for $G_{n}$ if and only if $\left\{e_{i}(x-c)\right\}_{i \in I}$ is an orthonormal basis for $\tilde{G}_{n}$. Since $P_{n} X_{j} P_{n}=0, \forall 1 \leq j \leq d$, we conclude that $\tilde{P}_{n}\left(X_{j}-c_{j}\right) \tilde{P}_{n}=0, \forall 1 \leq j \leq d$. Thus, for all $n \geq 0$, we have:

$$
\begin{aligned}
\left.\tilde{a}^{0}(j)\right|_{G_{n}} & =\tilde{P}_{n} X_{j} \tilde{P}_{n} \\
& =\tilde{P}_{n}\left(X_{j}-c_{j}\right) \tilde{P}_{n}+c_{j} \tilde{P}_{n} \\
& =c_{j} \tilde{P}_{n} .
\end{aligned}
$$

Hence $\tilde{a}^{0}(j)=c_{j} I$. Therefore, we obtain the following:

Theorem 3.2. If $\mu$ is a probability measure on $\mathbb{R}^{d}$, having finite moments of all orders, then $\mu$ is polynomially symmetric about the point $c=\left(c_{1}, c_{2}, \ldots, c_{d}\right) \in \mathbb{R}^{d}$ if and only if for all $j \in\{1,2, \ldots, d\}$, we have:

$$
a^{0}(j)=c_{j} I .
$$

\section{Polynomially Factorizable Measures}

Definition 4.1. If $\mu$ is a probability measure on the Borel subsets of $\mathbb{R}^{d}$, having finite moments of any order, then we say that $\mu$ is polynomially factorizable if for any non-negative integers $i_{1}, i_{2}, \ldots, i_{d}$ we have:

$$
E\left[x_{1}^{i_{1}} x_{2}^{i_{2}} \cdots x_{d}^{i_{d}}\right]=E\left[x_{1}^{i_{1}}\right] E\left[x_{2}^{i_{2}}\right] \cdots E\left[x_{d}^{i_{d}}\right] .
$$

In the above definition $E$ denotes the expectation with respect to $\mu$.

Observe that if $\mu$ is polynomially factorizable, then for any polynomial functions $f_{1}\left(x_{1}\right), f_{2}\left(x_{2}\right), \ldots, f_{d}\left(x_{d}\right)$, we have:

$$
E\left[f_{1}\left(x_{1}\right) f_{2}\left(x_{2}\right) \cdots f_{d}\left(x_{d}\right)\right]=E\left[f_{1}\left(x_{1}\right)\right] E\left[f_{2}\left(x_{2}\right)\right] \cdots E\left[f_{d}\left(x_{d}\right)\right] .
$$

If there exist $d$ probability measures $\mu_{1}, \mu_{2}, \ldots, \mu_{d}$ on $\mathbb{R}$, such that for all $B_{1}$, $B_{2}, \ldots, B_{d}$ Borel subsets of $\mathbb{R}$,

$$
\mu\left(B_{1} \times B_{2} \times \cdots \times B_{d}\right)=\mu_{1}\left(B_{1}\right) \mu_{2}\left(B_{2}\right) \cdots \mu_{d}\left(B_{d}\right),
$$


then $\mu$ is polynomially factorizable. The converse is not true. In Example 5.2 of Sec. 5 we present a polynomially factorizable measure that is not a product measure.

Let $\mu$ be a polynomially factorizable measure on the Borel subsets of $\mathbb{R}^{d}$. For any $i \in\{1,2, \ldots, d\}$, let $\mathcal{H}_{i}$ be the closure of the space $\mathcal{P}_{i}$, of all polynomial functions of the variable $x_{i}$, in the space $L^{2}\left(\mathbb{R}^{d}, \mu\right)$. Every function in $\mathcal{P}_{i}$ is a polynomial function depending only on the variable $x_{i}$. Let $\mathcal{H}$ be the closure of the space $\mathcal{P}$, of all polynomial functions of $d$ variables: $x_{1}, x_{2}, \ldots, x_{d}$, in the space $L^{2}\left(\mathbb{R}^{d}, \mu\right)$. The multilinear function $T: \mathcal{P}_{1} \times \mathcal{P}_{2} \times \cdots \times \mathcal{P}_{d} \rightarrow \mathcal{P}$, defined by $T\left(f_{1}, f_{2}, \ldots, f_{d}\right)=f$, where $f\left(x_{1}, x_{2}, \ldots, x_{d}\right)=f_{1}\left(x_{1}\right) f_{2}\left(x_{2}\right) \cdots f_{d}\left(x_{d}\right)$, generates a linear map $U$ between the algebraic tensor product $\mathcal{P}_{1} \otimes \mathcal{P}_{2} \otimes \cdots \otimes \mathcal{P}_{d}$ and the space $\mathcal{P}$. Because $\mu$ is polynomially factorizable, $U$ preserves the inner product. Indeed, for any $f_{1}, g_{1} \in$ $\mathcal{P}_{1}, f_{2}, g_{2} \in \mathcal{P}_{2}, \ldots, f_{d}, g_{d} \in \mathcal{P}_{d}$, we have:

$$
\begin{aligned}
\langle U( & \left.\left.f_{1} \otimes f_{2} \otimes \cdots \otimes f_{d}\right), U\left(g_{1} \otimes g_{2} \otimes \cdots \otimes g_{d}\right)\right\rangle \\
& =\left\langle f_{1}\left(x_{1}\right) f_{2}\left(x_{2}\right) \cdots f_{d}\left(x_{d}\right), g_{1}\left(x_{1}\right) g_{2}\left(x_{2}\right) \cdots g_{d}\left(x_{d}\right)\right\rangle \\
& =E\left[f_{1}\left(x_{1}\right) \bar{g}_{1}\left(x_{1}\right) f_{2}\left(x_{2}\right) \bar{g}_{2}\left(x_{2}\right) \cdots f_{d}\left(x_{d}\right) \bar{g}_{d}\left(x_{d}\right)\right] \\
& =E\left[f_{1}\left(x_{1}\right) \bar{g}_{1}\left(x_{1}\right)\right] E\left[f_{2}\left(x_{2}\right) \bar{g}_{2}\left(x_{2}\right)\right] \cdots E\left[f_{d}\left(x_{d}\right) \bar{g}_{d}\left(x_{d}\right)\right] \\
& =\left\langle f_{1}, g_{1}\right\rangle\left\langle f_{2}, g_{2}\right\rangle \cdots\left\langle f_{d}, g_{d}\right\rangle \\
& =\left\langle f_{1} \otimes f_{2} \otimes \cdots \otimes f_{d}, g_{1} \otimes g_{2} \otimes \cdots \otimes g_{d}\right\rangle .
\end{aligned}
$$

$U$ is onto since any monomial belongs to its range. Since $\mathcal{P}_{1} \otimes \mathcal{P}_{2} \otimes \cdots \otimes \mathcal{P}_{d}$ is dense in the Hilbert space $\mathcal{H}_{1} \otimes \mathcal{H}_{2} \otimes \cdots \otimes \mathcal{H}_{d}$ and $\mathcal{P}$ is dense in $\mathcal{H}$, the operator $U$ can be uniquely extended to a unitary operator $\tilde{U}$ from $\mathcal{H}_{1} \otimes \mathcal{H}_{2} \otimes \cdots \otimes \mathcal{H}_{d}$ onto $\mathcal{H}$. Thus we may identify the Hilbert spaces $\mathcal{H}_{1} \otimes \mathcal{H}_{2} \otimes \cdots \otimes \mathcal{H}_{d}$ and $\mathcal{H}$.

For any $i \in\{1,2, \ldots, d\}$ and $k$ a non-negative integer, let $F_{k}^{(i)}$ be the vector space spanned by the polynomial functions $1, x_{i}, x_{i}^{2}, \ldots, x_{i}^{k}$. Let $G_{k}^{(i)}$ be the orthogonal complement of $F_{k-1}^{(i)}$ into $F_{k}^{(i)}$, where $F_{-1}^{(i)}:=\{0\}$. To compute this orthogonal complement we consider $F_{k-1}^{(i)}$ and $F_{k}^{(i)}$ as being subspaces of the Hilbert space $\mathcal{H}_{i}$. We denote by $P_{k}^{(i)}$ the projection operator from $\mathcal{H}_{i}$ onto $G_{k}^{(i)}$ and by $X_{i}^{(i)}$ the densely defined operator on $\mathcal{H}_{i}$ given by the multiplication by the polynomial function $x_{i}$.

For any $i \in\{1,2, \ldots, d\}$ and $n$ a non-negative integer, we define the operators $D_{n, i}^{-}, D_{n, i}^{0}$ and $D_{n, i}^{+}$, from $\mathcal{H}_{i}$ into $\mathcal{H}_{i}$, in the following way:

$$
D_{n, i}^{-}=P_{n-1}^{(i)} X_{i}^{(i)} P_{n}^{(i)}, \quad D_{n, i}^{0}=P_{n}^{(i)} X_{i}^{(i)} P_{n}^{(i)} \quad \text { and } \quad D_{n, i}^{+}=P_{n+1}^{(i)} X_{i}^{(i)} P_{n}^{(i)} .
$$

Lemma 4.1. Let $\mu$ be a polynomially factorizable probability measure on the Borel subsets of $\mathbb{R}^{d}$. If we identify the space $\mathcal{H}$ with the space $\mathcal{H}_{1} \otimes \mathcal{H}_{2} \otimes \cdots \otimes \mathcal{H}_{d}$, then for any $n \geq 0$, we have:

$$
P_{n}=\sum_{i_{1}+i_{2}+\cdots+i_{d}=n} P_{i_{1}}^{(1)} \otimes P_{i_{2}}^{(2)} \otimes \cdots \otimes P_{i_{d}}^{(d)} .
$$

In the above sum all the indices $i_{j}$ are considered to be non-negative. 
Proof. Since the tensor product of orthogonal projections is an orthogonal projection, it follows that each term $P_{i_{1}}^{(1)} \otimes P_{i_{2}}^{(2)} \otimes \cdots \otimes P_{i_{d}}^{(d)}$ is an orthogonal projection. If $i_{j} \neq k_{j}$, then $P_{i_{j}}^{(j)} P_{k_{j}}^{(j)}=0$ and thus the terms in the sum from the right-hand side of (4.1) are orthogonal (i.e. the composition of any two different terms is zero). Thus the right-hand side of (4.1) is an orthogonal projection. If $P$ and $Q$ are two orthogonal projections of the same Hilbert space $H$, then we say that $P \geq Q$ if the range of $P$ contains the range of $Q$. If $i_{1}+i_{2}+\cdots+i_{d}=n$ and $f(x) \in F_{n-1}$, then $f(x)=\sum a_{j_{1}, j_{2}, \ldots, j_{d}} x_{1}^{j_{1}} x_{2}^{j_{2}} \cdots x_{d}^{j_{d}}$ with $j_{1}+j_{2}+\cdots+j_{d} \leq n-1$. Thus there exists $k \in\{1,2, \ldots, d\}$ such that $i_{k}>j_{k}$ and so $P_{i_{1}}^{(1)} \otimes \cdots \otimes P_{i_{d}}^{(d)}\left(x_{1}^{j_{1}} \cdots x_{d}^{j_{d}}\right)=0$. Hence $P_{i_{1}}^{(1)} \otimes \cdots \otimes P_{i_{d}}^{(d)} f(x)=0$. Therefore the range of $P_{i_{1}}^{(1)} \otimes \cdots \otimes P_{i_{d}}^{(d)}$ is orthogonal to the space $F_{n-1}$. It is obvious that the range of $P_{i_{1}}^{(1)} \otimes \cdots \otimes P_{i_{d}}^{(d)}$ is contained in $F_{n}$. Thus $P_{n} \geq P_{i_{1}}^{(1)} \otimes \cdots \otimes P_{i_{d}}^{(d)}$. Therefore $P_{n} \geq Q_{n}$, where $Q_{n}$ denotes the right-hand side of (4.1).

We prove now by induction on $n$, that $P_{n}=Q_{n}$. For $n=0$, this is obvious since both $P_{0}$ and $Q_{0}$ are projections on the one-dimensional vector space spanned by the vacuum vector 1 . Let us assume that $P_{k}=Q_{k}$, for all $k \leq n-1$, and prove that $P_{n}=Q_{n}$. To see this, we must show that for any monomial $m(x)=x^{i_{1}} \cdots x_{d}^{i_{d}}$, such that $i_{1}+\cdots+i_{d}=n$, we have $P_{n} m(x)=Q_{n} m(x)$. We can write:

$$
\begin{gathered}
x_{1}^{i_{1}}=P_{i_{1}}^{(1)} x_{1}^{i_{1}}+\sum_{j=0}^{i_{1}-1} P_{j}^{(1)} x_{1}^{i_{1}}, \\
\vdots \\
x_{d}^{i_{d}}=P_{i_{d}}^{(d)} x_{d}^{i_{d}}+\sum_{j=0}^{i_{d}-1} P_{j}^{(d)} x_{d}^{i_{d}} .
\end{gathered}
$$

Multiplying these relations and keeping in mind that we have identified $\mathcal{H}$ with $\mathcal{H}_{1} \otimes \mathcal{H}_{2} \otimes \cdots \otimes \mathcal{H}_{d}$, according to our induction bypothesis, we can see that:

$$
\begin{aligned}
P_{n} m(x) & =P_{i_{1}}^{(1)} x_{1}^{i_{1}} P_{i_{2}}^{(2)} x_{2}^{i_{2}} \cdots P_{i_{d}}^{(d)} x_{d}^{i_{d}} \\
& =\left(P_{i_{1}}^{(1)} \otimes P_{i_{2}}^{(2)} \otimes \cdots \otimes P_{i_{d}}^{(d)}\right)\left(x_{1}^{i_{1}} x_{2}^{i_{2}} \cdots x_{d}^{i_{d}}\right) \\
& =Q_{n} m(x) .
\end{aligned}
$$

Let $\mu$ be a polynomially factorizable measure on $\mathbb{R}^{d}$. If we identify the space $\mathcal{H}$ with the space $\mathcal{H}_{1} \otimes \mathcal{H}_{2} \otimes \cdots \otimes \mathcal{H}_{d}$, then for any $1 \leq j \leq d$, we have the operatorial relation:

$$
X_{j}=I_{1} \otimes \cdots \otimes I_{j-1} \otimes X_{j}^{(j)} \otimes I_{j+1} \otimes \cdots \otimes I_{d},
$$

where $I_{k}$ is the identity operator of the space $\mathcal{H}_{k}$, for any $k \neq j$.

Theorem 4.1. Let $\mu$ be a polynomially factorizable measure on $\mathbb{R}^{d}$. If we identify the space $\mathcal{H}$ with the space $\mathcal{H}_{1} \otimes \mathcal{H}_{2} \otimes \cdots \otimes \mathcal{H}_{d}$, then for all $1 \leq j \leq d$ and $n \geq 0$ 
the following three operatorial relations hold:

$$
\begin{aligned}
D_{n}^{-}(j) & =\sum_{i_{1}+\cdots+i_{d}=n} P_{i_{1}}^{(1)} \otimes \cdots \otimes P_{i_{j-1}}^{(j-1)} \otimes D_{i_{j}, j}^{-} \otimes P_{i_{j+1}}^{(j+1)} \otimes \cdots \otimes P_{i_{d}}^{(d)}, \\
D_{n}^{0}(j) & =\sum_{i_{1}+\cdots+i_{d}=n} P_{i_{1}}^{(1)} \otimes \cdots \otimes P_{i_{j-1}}^{(j-1)} \otimes D_{i_{j}, j}^{0} \otimes P_{i_{j+1}}^{(j+1)} \otimes \cdots \otimes P_{i_{d}}^{(d)}, \\
D_{n}^{+}(j) & =\sum_{i_{1}+\cdots+i_{d}=n} P_{i_{1}}^{(1)} \otimes \cdots \otimes P_{i_{j-1}}^{(j-1)} \otimes D_{i_{j}, j}^{+} \otimes P_{i_{j+1}}^{(j+1)} \otimes \cdots \otimes P_{i_{d}}^{(d)} .
\end{aligned}
$$

Proof. We will check only the first relation. The other two will be proved similarly. We have:

$$
\begin{aligned}
D_{n}^{-}(j) & \\
= & P_{n-1} X_{j} P_{n} \\
= & P_{n-1}\left(I_{1} \otimes \cdots \otimes X_{j}^{(j)} \otimes \cdots \otimes I_{d}\right) \\
& \times \sum_{i_{1}+\cdots+i_{d}=n} P_{i_{1}}^{(1)} \otimes \cdots \otimes P_{i_{j}}^{(j)} \otimes \cdots \otimes P_{i_{d}}^{(d)} \\
= & P_{n-1} \sum_{i_{1}+\cdots+i_{d}=n}\left(I_{1} P_{i_{1}}^{(1)}\right) \otimes \cdots \otimes\left(X_{j}^{(j)} P_{i_{j}}^{(j)}\right) \otimes \cdots \otimes\left(I_{d} P_{i_{d}}^{(d)}\right) \\
= & \sum_{k_{1}+\cdots+k_{d}=n-1} P_{k_{1}}^{(1)} \otimes \cdots \otimes P_{k_{j}}^{(j)} \otimes \cdots \otimes P_{k_{d}}^{(d)} \\
& \times \sum_{i_{1}+\cdots+i_{d}=n} P_{i_{1}}^{(1)} \otimes \cdots \otimes\left(X_{j}^{(j)} P_{i_{j}}^{(j)}\right) \otimes \cdots \otimes P_{i_{d}}^{(d)} \\
= & \sum_{k_{1}+\cdots+k_{d}=n-1} \sum_{i_{1}+\cdots+i_{d}=n}\left[\left(P_{k_{1}}^{(1)} P_{i_{1}}^{(1)}\right) \otimes \cdots \otimes\left(P_{k_{j}}^{(j)} X_{j}^{(j)} P_{i_{j}}^{(j)}\right)\right. \\
& \left.\otimes \cdots \otimes\left(P_{k_{d}}^{(d)} P_{i_{d}}^{(d)}\right)\right],
\end{aligned}
$$

where " $X$ " means composition of operators.

Observe that if

$$
\left(P_{k_{1}}^{(1)} P_{i_{1}}^{(1)}\right) \otimes \cdots \otimes\left(P_{k_{j}}^{(j)} X_{j}^{(j)} P_{i_{j}}^{(j)}\right) \otimes \cdots \otimes\left(P_{k_{d}}^{(d)} P_{i_{d}}^{(d)}\right) \neq 0,
$$

then $i_{1}=k_{1}, \ldots, i_{j-1}=k_{j-1}, i_{j+1}=k_{j+1}, \ldots, i_{d}=k_{d}$. Since $i_{1}+\cdots+i_{d}=n$ and $k_{1}+\cdots+k_{d}=n-1$, it follows that $i_{j}=k_{j}+1$. Thus we obtain:

$$
\begin{aligned}
D_{n}^{-}(j) & =\sum_{i_{1}+\cdots+i_{d}=n} P_{i_{1}}^{(1)} \otimes \cdots \otimes\left(P_{i_{j}-1}^{(j)} X_{j}^{(j)} P_{i_{j}}^{(j)}\right) \otimes \cdots \otimes P_{i_{d}}^{(d)} \\
& =\sum_{i_{1}+\cdots+i_{d}=n} P_{i_{1}}^{(1)} \otimes \cdots \otimes D_{i_{j}, j}^{-} \otimes \cdots \otimes P_{i_{d}}^{(d)} .
\end{aligned}
$$


Theorem 4.2. Let $\mu$ be a polynomially factorizable probability measure on the Borel subsets of $\mathbb{R}^{d}$. Then, for all $j \neq k$, we have:

$$
\begin{aligned}
& a^{-}(j) a^{+}(k)=a^{+}(k) a^{-}(j), \\
& a^{0}(j) a^{+}(k)=a^{+}(k) a^{0}(j), \\
& a^{0}(j) a^{-}(k)=a^{-}(k) a^{0}(j) .
\end{aligned}
$$

Proof. To check the commutation relation $a^{-}(j) a^{+}(k)=a^{+}(k) a^{-}(j)$, we need to check that for all $n \geq 0$, we have $D_{n+1}^{-}(j) D_{n}^{+}(k)=D_{n-1}^{+}(k) D_{n}^{-}(j)$. Let us assume that $j<k$. The case $j>k$ can be treated similarly or by duality. From the previous theorem we know that:

$$
\begin{aligned}
& D_{n+1}^{-}(j) D_{n}^{+}(k) \\
& =\sum_{r_{1}+\cdots+r_{d}=n+1} P_{r_{1}}^{(1)} \otimes \cdots \otimes P_{r_{j-1}}^{(j-1)} \otimes D_{r_{j}, j}^{-} \otimes P_{r_{j+1}}^{(j+1)} \otimes \cdots \otimes P_{r_{d}}^{(d)} \\
& \sum_{s_{1}+\cdots+s_{d}=n} P_{s_{1}}^{(1)} \otimes \cdots \otimes P_{s_{k-1}}^{(k-1)} \otimes D_{s_{k}, k}^{+} \otimes P_{s_{k+1}}^{(k+1)} \otimes \cdots \otimes P_{s_{d}}^{(d)} \\
& =\sum_{r_{1}+\cdots+r_{d}=n+1} \sum_{s_{1}+\cdots+s_{d}=n}\left[\left(P_{r_{1}}^{(1)} P_{s_{1}}^{(1)}\right) \otimes \cdots \otimes\left(D_{r_{j}, j}^{-} P_{s_{j}}^{(j)}\right)\right. \\
& \left.\otimes \cdots \otimes\left(P_{r_{k}}^{(k)} D_{s_{k}, k}^{+}\right) \otimes \cdots \otimes\left(P_{r_{d}}^{(d)} P_{s_{d}}^{(d)}\right)\right] .
\end{aligned}
$$

To have $P_{r_{l}}^{(l)} P_{s_{l}}^{(l)} \neq 0$, for all $l \in\{1, \ldots, d\} \backslash\{j, k\}$, we must have $r_{l}=s_{l}$, for all $l \in\{1, \ldots, d\} \backslash\{j, k\}$.

Since $D_{r_{j}, j}^{-} P_{s_{j}}^{(j)}=P_{r_{j}-1}^{(j)} X_{j}^{(j)} P_{r_{j}}^{(j)} P_{s_{j}}^{(j)}$, if $D_{r_{j}, j}^{-} P_{s_{j}}^{(j)} \neq 0$, then $r_{j}=s_{j}$. If $r_{j}=s_{j}$, then $D_{r_{j}, j}^{-} P_{s_{j}}^{(j)}=D_{s_{j}, j}^{-}$.

Since $P_{r_{k}}^{(k)} D_{s_{k}, k}^{+}=P_{r_{k}}^{(k)} P_{s_{k}+1}^{(k)} X_{k}^{(k)} P_{s_{k}}^{(k)}$, if $P_{r_{k}}^{(k)} D_{s_{k}, k}^{+} \neq 0$, then $r_{k}=s_{k}+1$. If $r_{k}=s_{k}+1$, then $P_{r_{k}}^{(k)} D_{s_{k}, k}^{+}=D_{s_{k}, k}^{+}$.

Thus we obtain:

$$
\begin{aligned}
& D_{n+1}^{-}(j) D_{n}^{+}(k) \\
& \quad=\sum_{s_{1}+\cdots+s_{d}=n} P_{s_{1}}^{(1)} \otimes \ldots \otimes D_{s_{j}, j}^{-} \otimes \cdots \otimes D_{s_{k}, k}^{+} \otimes \cdots \otimes P_{s_{d}}^{(d)} .
\end{aligned}
$$

In the same way, we can show that:

$$
\begin{aligned}
& D_{n-1}^{+}(k) D_{n}^{-}(j) \\
& \quad=\sum_{s_{1}+\cdots+s_{d}=n} P_{s_{1}}^{(1)} \otimes \cdots \otimes D_{s_{j}, j}^{-} \otimes \cdots \otimes D_{s_{k}, k}^{+} \otimes \cdots \otimes P_{s_{d}}^{(d)} .
\end{aligned}
$$

Thus $D_{n+1}^{-}(j) D_{n}^{+}(k)=D_{n-1}^{+}(k) D_{n}^{-}(j)$.

The commutation relationships (4.4) and (4.5) are proved similarly. 
Proposition 4.1. If $\mu$ is a probability measure on $\mathbb{R}^{d}$ such that for any $j \neq k$, the operators $a^{-}(j)$ and $a^{+}(k)$ commute, then for any $j, k \in\{1,2, \ldots, d\}$ we have:

$$
a^{0}(j) a^{0}(k)=a^{0}(k) a^{0}(j) .
$$

Proof. Let $j, k \in\{1,2, \ldots, d\}$ be fixed.

If $j=k$, then it is obvious that $a^{0}(j)$ and $a^{0}(k)$ commute.

If $j \neq k$, then, according to Eq. (2.10), for any $n \geq 0$, we have:

$$
\begin{aligned}
& D_{n+1}^{-}(j) D_{n}^{+}(k)+D_{n}^{0}(j) D_{n}^{0}(k)+D_{n-1}^{+}(j) D_{n}^{-}(k) \\
& \quad=D_{n+1}^{-}(k) D_{n}^{+}(j)+D_{n}^{0}(k) D_{n}^{0}(j)+D_{n-1}^{+}(k) D_{n}^{-}(j) .
\end{aligned}
$$

Since $a^{-}(j) a^{+}(k)=a^{+}(k) a^{-}(j)$ it follows that

$$
D_{n+1}^{-}(j) D_{n}^{+}(k)=D_{n-1}^{+}(k) D_{n}^{-}(j) .
$$

Since $a^{+}(j) a^{-}(k)=a^{-}(k) a^{+}(j)$ it follows that

$$
D_{n-1}^{+}(j) D_{n}^{-}(k)=D_{n+1}^{-}(k) D_{n}^{+}(j) .
$$

Thus we obtain

$$
D_{n}^{0}(j) D_{n}^{0}(k)=D_{n}^{0}(k) D_{n}^{0}(j) .
$$

Since this is true for all $n \geq 1$, we obtain $a^{0}(j) a^{0}(k)=a^{0}(k) a^{0}(j)$.

Proposition 4.2. Let $\mu$ be a probability measure on $\mathbb{R}^{d}$ having finite moments of any order. Let $j, k \in\{1,2, \ldots, d\}$. Then the following two statements are equivalent:

(1) $a^{0}(j) a^{+}(k)=a^{+}(k) a^{0}(j)$ and $a^{0}(j) a^{0}(k)=a^{0}(k) a^{0}(j)$.

(2) $a^{0}(j) X_{k}=X_{k} a^{0}(j)$.

Proof. $(1) \Rightarrow(2)$ Let us assume that $a^{0}(j) a^{+}(k)=a^{+}(k) a^{0}(j)$ and $a^{0}(j) a^{0}(k)=$ $a^{0}(k) a^{0}(j)$. Taking the adjoints on both sides of the first equality we obtain $a^{-}(k) a^{0}(j)=a^{0}(j) a^{-}(k)$. Thus:

$$
\begin{aligned}
a^{0}(j) X_{k} & =a^{0}(j)\left[a^{+}(k)+a^{0}(k)+a^{-}(k)\right] \\
& =a^{0}(j) a^{+}(k)+a^{0}(j) a^{0}(k)+a^{0}(j) a^{-}(k) \\
& =a^{+}(k) a^{0}(j)+a^{0}(k) a^{0}(j)+a^{-}(k) a^{0}(j) \\
& =\left[a^{+}(k)+a^{0}(k)+a^{-}(k)\right] a^{0}(j) \\
& =X_{k} a^{0}(j) .
\end{aligned}
$$

$(2) \Rightarrow(1)$ Let us assume now that $a^{0}(j) X_{k}=X_{k} a^{0}(j)$. Let $n \geq 0$ be fixed. For any $\varphi \in G_{n}$ we have $a^{0}(j) X_{k} \varphi=X_{k} a^{0}(j) \varphi$. This means

$$
\begin{aligned}
& a^{0}(j) a^{+}(k) \varphi+a^{0}(j) a^{0}(k) \varphi+a^{0}(j) a^{-}(k) \varphi \\
& \quad=a^{+}(k) a^{0}(j) \varphi+a^{0}(k) a^{0}(j) \varphi+a^{-}(k) a^{0}(j) \varphi .
\end{aligned}
$$


Since $a^{0}(j) a^{+}(k) \varphi \in G_{n+1}$ and $a^{+}(k) a^{0}(j) \varphi \in G_{n+1}, a^{0}(j) a^{0}(k) \varphi \in G_{n}$ $a^{0}(k) a^{0}(j) \varphi \in G_{n}, a^{0}(j) a^{-}(k) \varphi \in G_{n-1}$ and $a^{-}(k) a^{0}(j) \varphi \in G_{n-1}$, and the spaces $G_{n+1}, G_{n}$ and $G_{n-1}$ are orthogonal, we obtain: $a^{0}(j) a^{+}(k) \varphi=a^{+}(k) a^{0}(j) \varphi$, $a^{0}(j) a^{0}(k) \varphi=a^{0}(k) a^{0}(j) \varphi$ and $a^{0}(j) a^{-}(k) \varphi=a^{-}(k) a^{0}(j) \varphi$.

Combining Theorem 4.2, Proposition 4.1, and Theorem 2.3 we obtain the following:

Corollary 4.1. Let $\mu$ be a polynomially factorizable probability measure on the Borel subsets of $\mathbb{R}^{d}$. If $j, k \in\{1,2, \ldots, d\}$ and $j \neq k$, then for any $Y \in$ $\left\{a^{-}(j), a^{0}(j), a^{+}(j)\right\}$ and $Z \in\left\{a^{-}(k), a^{0}(k), a^{+}(k)\right\}$, we have:

$$
Y Z=Z Y \text {. }
$$

Theorem 4.3. If $\mu$ is a polynomially factorizable probability measure on the Borel subsets of $\mathbb{R}^{d}$, then for all $i, j, k \in\{1,2, \ldots, d\}$, we have:

$$
\left[a^{0}(i),\left[a^{-}(j), a^{+}(k)\right]\right]=0,
$$

where $[A, B]:=A B-B A$ for any two operators $A$ and $B$.

Proof. We analyze three cases:

Case I. If $j \neq k$, then Theorem 4.2 implies $\left[a^{-}(j), a^{+}(k)\right]=0$. Thus $\left[a^{0}(i),\left[a^{-}(j), a^{+}(k)\right]\right]=0$.

Case II. If $j=k$ and $i \neq j$, then according to Theorem $4.2, a^{0}(i)$ commutes with both operators $a^{-}(j)$ and $a^{+}(j)$. Thus $a^{0}(i)$ commutes with the commutator of $a^{-}(j)$ and $a^{+}(j)$.

Case III. If $i=j=k$, then according to Theorem 4.1, for any $n \geq 0$, we have:

$a^{0}(j)\left[a^{-}(j), a^{+}(j)\right]_{\mid G_{n}}$

$$
=\sum_{l_{1}+\cdots+l_{d}=n} P_{l_{1}}^{(1)} \otimes \cdots \otimes D_{l_{j}, j}^{0}\left(D_{l_{j}+1, j}^{-} D_{l_{j}, j}^{+}-D_{l_{j}-1, j}^{+} D_{l_{j}, j}^{-}\right) \otimes \cdots \otimes P_{l_{d}}^{(d)} .
$$

We also have:

$$
\begin{aligned}
& {\left[a^{-}(j), a^{+}(j)\right] a^{0}(j)_{\mid G_{n}}} \\
& \quad=\sum_{l_{1}+\cdots+l_{d}=n} P_{l_{1}}^{(1)} \otimes \cdots \otimes\left(D_{l_{j}+1, j}^{-} D_{l_{j}, j}^{+}-D_{l_{j}-1, j}^{+} D_{l_{j}, j}^{-}\right) D_{l_{j}, j}^{0} \otimes \cdots \otimes P_{l_{d}}^{(d)} .
\end{aligned}
$$

$D_{l_{j}, j}^{0}$ and $D_{l_{j}+1, j}^{-} D_{l_{j}, j}^{+}-D_{l_{j}-1, j}^{+} D_{l_{j}, j}^{-}$are linear operators from the space $G_{n}^{(j)}$ into itself. Since the vector space $G_{n}^{(j)}$ has dimension at most 1, any two linear operators from $G_{n}^{(j)}$ into $G_{n}^{(j)}$ commute. Thus, we have:

$$
D_{l_{j}, j}^{0}\left(D_{l_{j}+1, j}^{-} D_{l_{j}, j}^{+}-D_{l_{j}-1, j}^{+} D_{l_{j}, j}^{-}\right)=\left(D_{l_{j}+1, j}^{-} D_{l_{j}, j}^{+}-D_{l_{j}-1, j}^{+} D_{l_{j}, j}^{-}\right) D_{l_{j}, j}^{0} .
$$

Therefore, we obtain:

$$
a^{0}(j)\left[a^{-}(j), a^{+}(j)\right]_{\mid G_{n}}=\left[a^{-}(j), a^{+}(j)\right] a^{0}(j)_{\mid G_{n}},
$$

for all $n \geq 0$. Thus $\left[a^{0}(j),\left[a^{-}(j), a^{+}(j)\right]\right]=0$. 
We prove now the converse of Theorem 4.2.

Theorem 4.4. Let $\mu$ be a probability measure on the Borel subsets of $\mathbb{R}^{d}$ such that, for all $j \neq k$, we have:

$$
\begin{aligned}
a^{-}(j) a^{+}(k) & =a^{+}(k) a^{-}(j), \\
a^{0}(j) a^{+}(k) & =a^{+}(k) a^{0}(j) .
\end{aligned}
$$

Then $\mu$ is polynomially factorizable.

Proof. Since for all $j \neq k, a^{0}(j) a^{+}(k)=a^{+}(k) a^{0}(j)$, taking the adjoints on both sides of this equation, we obtain: $a^{-}(k) a^{0}(j)=a^{0}(j) a^{-}(k)$. Because for all $j \neq k$, the operators $a^{-}(j)$ and $a^{+}(k)$ commute, according to Proposition 4.1, for any $r$, $s \in\{1,2, \ldots, d\}$, the operators $a^{0}(r)$ and $a^{0}(s)$ also commute. Using Theorem 2.3, we conclude that, for all $j \neq k$, any operator from the set $\left\{a^{-}(j), a^{0}(j), a^{+}(j)\right\}$ commutes with any operator from the set $\left\{a^{-}(k), a^{0}(k), a^{+}(k)\right\}$.

For any $1 \leq j \leq d$ and $n \geq 1$, we define a $j$-word of length $n$, to be any operator of the form $w=a^{\varepsilon_{1}}(j) a^{\varepsilon_{2}}(j) \cdots a^{\varepsilon_{n}}(j)$, where $\varepsilon_{i} \in\{-, 0,+\}, \forall 1 \leq i \leq n$. The operators $a^{\varepsilon_{1}}(j), a^{\varepsilon_{2}}(j), \ldots, a^{\varepsilon_{n}}(j)$ are called the letters of the word $w$.

The identity operator $I$, of $L^{2}\left(\mathbb{R}^{d}, \mu\right)$, is considered to be a $j$-word of length 0 , for any $1 \leq j \leq d$.

Let $m(x)=x_{1}^{i_{1}} x_{2}^{i_{2}} \cdots x_{d}^{i_{d}}$ be a monomial. Let $\phi=1$ be the vacuum vector (the constant polynomial function 1 ). We have

$$
\begin{aligned}
m(x) & =X_{1}^{i_{1}} X_{2}^{i_{2}} \cdots X_{d}^{i_{d}} \phi \\
& =\left(a^{-}(1)+a^{0}(1)+a^{+}(1)\right)^{i_{1}} \cdots\left(a^{-}(d)+a^{0}(d)+a^{+}(d)\right)^{i_{d}} \phi \\
& =\sum\left(a^{\varepsilon_{1}^{1}}(1) a^{\varepsilon_{2}^{1}}(1) \cdots a^{\varepsilon_{i_{1}}^{1}}(1)\right) \cdots\left(a^{\varepsilon_{1}^{d}}(d) a^{\varepsilon_{2}^{d}}(d) \cdots a^{\varepsilon_{i_{d}}^{d}}(d)\right) \phi,
\end{aligned}
$$

where $\varepsilon_{r}^{s} \in\{-, 0,+\}$, for all $1 \leq s \leq d$ and $1 \leq r \leq i_{s}$. Thus

$$
m(x)=\sum w_{1} w_{2} \cdots w_{d} \phi,
$$

where $w_{j}$ is a $j$-word of length $i_{j}, \forall 1 \leq j \leq d$. According to our hypothesis, if $j \neq k$, then any $j$-word commutes with any $k$-word.

Since for any $1 \leq j \leq d$ and any integer $n, a^{-}(j)_{\mid F_{n}}: F_{n} \rightarrow F_{n-1}$, we say that $a^{-}(j)$ represents one step backward. Similarly we say that $a^{+}(j)$ represents one step forward, while $a^{0}(j)$ represents a neutral step. Thus $a^{-}(j)$ is considered to be a negative letter, $a^{+}(j)$ a positive letter, and $a^{0}(j)$ a neutral letter. If $n$ is a negative integer, then we declare $F_{n}$ to be the null space $\{0\}$. We define the signum $s(w)$ of a word $w$, to be the number of positive letters of $w$ minus the number of negative letters of $w$. If $w^{*}$ denotes the adjoint of $w$, then $s\left(w^{*}\right)=-s(w)$.

All the terms $w_{1} w_{2} \cdots w_{d} \phi$, containing at least one word $w_{j_{0}}$ that has more negative letters than positive letters, are equal to 0 . Indeed, for such a term,

$$
w_{1} w_{2} \cdots w_{d} \phi=w_{1} \cdots w_{j_{0}-1} w_{j_{0}+1} \cdots w_{d}\left(w_{j_{0}} \phi\right)=0
$$


since $w_{j_{0}} \phi=0$, because we start from the vacuum space and do more steps backward than we do forward. Thus $m(x)=\sum w_{1} w_{2} \cdots w_{d} \phi$, where each term contains only words having the number of negative letters less than or equal to the number of positive letters. Hence:

$$
\begin{aligned}
E[m(x)] & =\langle m(x), \phi\rangle \\
& =\sum_{s\left(w_{1}\right) \geq 0, \ldots, s\left(w_{d}\right) \geq 0}\left\langle w_{1} \cdots w_{d} \phi, \phi\right\rangle \\
& =\sum_{s\left(w_{1}\right) \geq 0, \ldots, s\left(w_{d}\right) \geq 0}\left\langle\phi, w_{d}^{*} \cdots w_{1}^{*} \phi\right\rangle .
\end{aligned}
$$

Observe that in the last sum all the terms, for which at least one of the words $\left\{w_{j}\right\}_{1 \leq j \leq d}$ has a positive signum, are equal to zero. This is true, because if there exists $j \in\{1,2, \ldots, d\}$ such that $s\left(w_{j}\right)>0$, then $s\left(w_{j}^{*}\right)<0$ and, it follows, as before, that $w_{d}^{*} \cdots w_{1}^{*} \phi=0$. Therefore, we have:

$$
E[m(x)]=\sum_{s\left(w_{1}\right)=0, \ldots, s\left(w_{d}\right)=0}\left\langle w_{1} \cdots w_{d} \phi, \phi\right\rangle .
$$

Observe that, since $s\left(w_{d}\right)=0, w_{d} \phi \in F_{0}$, and thus $w_{d} \phi=P_{0}\left(w_{d} \phi\right)=\left\langle w_{d} \phi, \phi\right\rangle \phi=$ $E\left[w_{d} \phi\right] \phi$. Applying $w_{d-1}$ to both sides of the equality: $w_{d} \phi=E\left[w_{d} \phi\right] \phi$, we get $w_{d-1} w_{d} \phi=E\left[w_{d} \phi\right] E\left[w_{d-1} \phi\right] \phi$. Iterating this process we obtain finally:

$$
w_{1} \cdots w_{d} \phi=E\left[w_{d} \phi\right] \cdots E\left[w_{1} \phi\right] \phi .
$$

Thus we obtain:

$$
\begin{aligned}
E[m(x)]= & \sum_{s\left(w_{1}\right)=0, s\left(w_{2}\right)=0, \ldots, s\left(w_{d}\right)=0}\left\langle w_{1} w_{2} \cdots w_{d} \phi, \phi\right\rangle \\
= & \sum_{s\left(w_{1}\right)=0, s\left(w_{2}\right)=0, \ldots, s\left(w_{d}\right)=0}\left\langle E\left[w_{d} \phi\right] E\left[w_{d-1} \phi\right] \cdots E\left[w_{1} \phi\right] \phi, \phi\right\rangle \\
= & \sum_{s\left(w_{1}\right)=0, s\left(w_{2}\right)=0, \ldots, s\left(w_{d}\right)=0} E\left[w_{d} \phi\right] E\left[w_{d-1} \phi\right] \cdots E\left[w_{1} \phi\right]\langle\phi, \phi\rangle \\
= & \sum_{s\left(w_{1}\right)=0, s\left(w_{2}\right)=0, \ldots, s\left(w_{d}\right)=0} E\left[w_{1} \phi\right] E\left[w_{2} \phi\right] \cdots E\left[w_{d} \phi\right] \\
= & \sum_{s\left(w_{1}\right)=0} E\left[w_{1} \phi\right] \sum_{s\left(w_{2}\right)=0} E\left[w_{2} \phi\right] \cdots \sum_{s\left(w_{d}\right)=0} E\left[w_{d} \phi\right] .
\end{aligned}
$$

Applying the last equality to the particular monomials $m_{1}(x)=x_{1}^{i_{1}}, m_{2}(x)=$ $x_{2}^{i_{2}}, \ldots, m_{d}(x)=x_{d}^{i_{d}}$, we can see that: $E\left[x_{1}^{i_{1}}\right]=\sum_{s\left(w_{1}\right)=0} E\left[w_{1} \phi\right], E\left[x_{2}^{i_{2}}\right]=$ $\sum_{s\left(w_{2}\right)=0} E\left[w_{2} \phi\right], \ldots, E\left[x_{d}^{i_{d}}\right]=\sum_{s\left(w_{d}\right)=0} E\left[w_{d} \phi\right]$. Thus:

$$
E[m(x)]=E\left[x_{1}^{i_{1}}\right] E\left[x_{2}^{i_{2}}\right] \cdots E\left[x_{d}^{i_{d}}\right] .
$$

Therefore $\mu$ is polynomially factorizable. 
From Proposition 4.2, Theorem 4.2 and Theorem 4.4 we obtain the following:

Theorem 4.5. A probability measure $\mu$ on $\mathbb{R}^{d}$, having finite moments of any order, is polynomially factorizable if and only if for all $j, k \in\{1,2, \ldots, d\}$, such that $j \neq k$, the commutators $\left[a^{-}(j), a^{+}(k)\right]$ and $\left[a^{0}(j), X_{k}\right]$ are both equal to zero.

Corollary 4.2. A polynomially symmetric about a point probability measure on $\mathbb{R}^{d}$ is polynomially factorizable if and only if for all $j \neq k$ we have $a^{-}(j) a^{+}(k)=$ $a^{+}(k) a^{-}(j)$.

\section{Examples}

In this section we will give an example of a polynomially symmetric probability measure that is not symmetric and an example of a polynomially factorizable probability measure that is not a product measure.

Let us consider the following function, introduced by Stieltjes in Ref. 12: $f$ : $(0, \infty) \rightarrow \mathbb{R}, f(t)=t^{-\ln t} \sin (2 \pi \ln t)$.

Claim 5.1. For all non-negative integer $n$, we have:

$$
\int_{0}^{\infty} t^{n} f(t) d t=0
$$

We present below the proof of this claim as it appears in Ref. 11. Indeed, for any non-negative integer $n$, making the change of variable $t=e^{u+\frac{n+1}{2}}$, we have:

$$
\begin{aligned}
\int_{0}^{\infty} & t^{n} f(t) d t \\
& =\int_{0}^{\infty} t^{n} t^{-\ln t} \sin (2 \pi \ln t) d t \\
& =\int_{\mathbb{R}} e^{n\left(u+\frac{n+1}{2}\right)} e^{-\left(u+\frac{n+1}{2}\right)^{2}} \sin \left(2 \pi\left(u+\frac{n+1}{2}\right)\right) e^{u+\frac{n+1}{2}} d u \\
& =e^{\left(\frac{n+1}{2}\right)^{2}} \int_{\mathbb{R}} e^{-u^{2}}[\sin (2 \pi u) \cos ((n+1) \pi)+\cos (2 \pi u) \sin ((n+1) \pi)] d u \\
& =(-1)^{n+1} e^{\left(\frac{n+1}{2}\right)^{2}} \int_{\mathbb{R}} e^{-u^{2}} \sin (2 \pi u) d u \\
& =0 .
\end{aligned}
$$

The last integral is zero since the integrand is an odd function.

Example 5.1. Let $\mu$ be the probability measure on $\mathbb{R}$ given by the density function $g: \mathbb{R} \rightarrow[0, \infty)$, defined by:

$$
g(x)= \begin{cases}c f^{+}(x) & \text { if } x>0 \\ 0 & \text { if } x=0 \\ c f^{-}(-x) & \text { if } x<0\end{cases}
$$


where $a^{+}=\max (a, 0), a^{-}=-\min (a, 0), \forall a \in \mathbb{R}$, and $c$ is a positive constant chosen such that $\int_{\mathbb{R}} g(x) d x=1$.

It is easy to see that $\mu$ has finite moments of any order.

Claim 5.2. $\mu$ is polynomially symmetric.

Indeed, if $n$ is an odd natural number, then we have:

$$
\begin{aligned}
\int_{\mathbb{R}} x^{n} \mu(d x) & =\int_{\mathbb{R}} x^{n} g(x) d x \\
& =c \int_{-\infty}^{0} x^{n} f^{-}(-x) d x+c \int_{0}^{\infty} x^{n} f^{+}(x) d x \\
& =c \int_{0}^{\infty}(-t)^{n} f^{-}(t) d t+c \int_{0}^{\infty} t^{n} f^{+}(t) d t \\
& =-c \int_{0}^{\infty} t^{n} f^{-}(t) d t+c \int_{0}^{\infty} t^{n} f^{+}(t) d t \\
& =c \int_{0}^{\infty} t^{n}\left[f^{+}(t)-f^{-}(t)\right] d t \\
& =c \int_{0}^{\infty} t^{n} f(t) d t \\
& =0
\end{aligned}
$$

Claim 5.3. $\mu$ is not symmetric.

Indeed, for any interval $[a, b]$ contained in the set: $\{x>0 \mid f(x)>0\}$ we have $\int_{a}^{b} g(x) d x=c \int_{a}^{b} f^{+}(x) d x>0$, but $\int_{-b}^{-a} g(x) d x=c \int_{a}^{b} f^{-}(x) d x=0$. Thus $\mu([a, b]) \neq \mu([-b,-a])$.

Let $f_{1}:(0, \infty) \rightarrow[0, \infty), f_{1}(t)=t^{-\ln t}[1+\sin (2 \pi \ln t)]$ and $f_{2}:(0, \infty) \rightarrow[0, \infty)$, $f_{2}(t)=t^{-\ln t}[1-\sin (2 \pi \ln t)]$. Since, according to (5.1), $\int_{0}^{\infty} t^{n} t^{-\ln t} \sin (2 \pi \ln t) d t=$ 0 , for any non-negative integer $n$, we have:

$$
\int_{0}^{\infty} t^{n} f_{1}(t) d t=\int_{0}^{\infty} t^{n} f_{2}(t) d t, \quad \forall n \in \mathbb{N} \cup\{0\} .
$$

In particular for $n=0$, let $k:=\int_{0}^{\infty} f_{1}(t) d t=\int_{0}^{\infty} f_{2}(t) d t$.

Example 5.2. Let $\mu$ be the probability measure on $\mathbb{R}^{2}$ given by the density function $h: \mathbb{R}^{2} \rightarrow[0, \infty)$, defined by:

$$
h(x, y)= \begin{cases}\frac{1}{k}\left[f_{1}(x) \sin ^{2} y+f_{2}(x) \cos ^{2} y\right] e^{-y} & \text { if } x>0 \text { and } y>0 \\ 0 & \text { otherwise }\end{cases}
$$


For any non-negative integers $m$ and $n$, we have:

$$
\begin{aligned}
\int_{\mathbb{R}^{2}} & x^{n} y^{m} \mu(d x d y) \\
& =\frac{1}{k} \int_{0}^{\infty} y^{m} e^{-y}\left[\sin ^{2} y \int_{0}^{\infty} x^{n} f_{1}(x) d x+\cos ^{2} y \int_{0}^{\infty} x^{n} f_{2}(x) d x\right] d y \\
& =\frac{1}{k} \int_{0}^{\infty} y^{m} e^{-y}\left[\int_{0}^{\infty} x^{n} f_{1}(x) d x\right]\left(\sin ^{2} y+\cos ^{2} y\right) d y \\
& =\frac{1}{k}\left[\int_{0}^{\infty} x^{n} f_{1}(x) d x\right] \int_{0}^{\infty} y^{m} e^{-y} d y .
\end{aligned}
$$

Thus we obtain:

$$
\int_{\mathbb{R}^{2}} x^{n} y^{m} \mu(d x d y)=\frac{1}{k}\left[\int_{0}^{\infty} x^{n} f_{1}(x) d x\right] \int_{0}^{\infty} y^{m} e^{-y} d y .
$$

In particular for $m=n=0$, we obtain:

$$
\begin{aligned}
\int_{\mathbb{R}^{2}} 1 \mu(d x d y) & =\frac{1}{k}\left[\int_{0}^{\infty} f_{1}(x) d x\right] \int_{0}^{\infty} e^{-y} d y \\
& =\frac{1}{k} \cdot k \cdot 1 \\
& =1 .
\end{aligned}
$$

Hence $\mu$ is a probability measure on $\mathbb{R}^{2}$.

Taking $m=0$ in formula (5.3), we can see that:

$$
\begin{aligned}
\int_{\mathbb{R}^{2}} x^{n} \mu(d x d y) & =\frac{1}{k} \int_{0}^{\infty} x^{n} f_{1}(x) d x \int_{0}^{\infty} e^{-y} d y \\
& =\frac{1}{k} \int_{0}^{\infty} x^{n} f_{1}(x) d x
\end{aligned}
$$

Hence

$$
\int_{\mathbb{R}^{2}} x^{n} \mu(d x d y)=\frac{1}{k} \int_{0}^{\infty} x^{n} f_{1}(x) d x .
$$

Taking $n=0$ in formula (5.3), we can see that:

$$
\begin{aligned}
\int_{\mathbb{R}^{2}} y^{m} \mu(d x d y) & =\frac{1}{k} \int_{0}^{\infty} f_{1}(x) d x \int_{0}^{\infty} y^{m} e^{-y} d y \\
& =\frac{1}{k} \cdot k \cdot \int_{0}^{\infty} y^{m} e^{-y} d y \\
& =\int_{0}^{\infty} y^{m} e^{-y} d y .
\end{aligned}
$$


Hence

$$
\int_{\mathbb{R}^{2}} y^{m} \mu(d x d y)=\int_{0}^{\infty} y^{m} e^{-y} d y .
$$

From formulas (5.3)-(5.5), we can see that:

$$
\int_{\mathbb{R}^{2}} x^{n} y^{m} \mu(d x d y)=\int_{0}^{\infty} x^{n} \mu(d x d y) \int_{0}^{\infty} y^{m} \mu(d x d y) .
$$

Thus $\mu$ is polynomially factorizable.

Claim 5.4. $\mu$ is not a product measure.

Let us assume that two probability measures, $\mu_{1}$ and $\mu_{2}$, on $\mathbb{R}$, exist, such that for any two Borel subsets $B_{1}$ and $B_{2}$ of $\mathbb{R}$, we have $\mu\left(B_{1} \times B_{2}\right)=\mu_{1}\left(B_{1}\right) \mu_{2}\left(B_{2}\right)$. Since $\mu$ is absolutely continuous with respect to the Lebesgue measure on $\mathbb{R}^{2}$, it follows that $\mu_{1}$ and $\mu_{2}$ are absolutely continuous with respect to the Lebesgue measure on $\mathbb{R}$. If $u$ and $v$ are the density functions of $\mu_{1}$ and $\mu_{2}$, respectively, then we must have for almost all $(x, y) \in \mathbb{R}^{2}, h(x, y)=u(x) v(y)$, which is impossible since $h(x, y)$ cannot be written as a function of $x$ times a function of $y$.

\section{Acknowledgments}

This research was carried out during the visits of H.-H. Kuo and A. Stan to the Centro Vito Volterra (CVV), Università di Roma "Tor Vergata", May-June (H.H.K.) and June (A.S.) 2002. We would like to thank CVV for financial support and Professor L. Accardi for the warm hospitality during our visits. A. S. was partially supported by the NSF grant DMS-9972961.

\section{References}

1. L. Accardi and M. Bożejko, Interacting Fock space and Gaussianization of probability measures, Infin. Dim. Anal. Quantum Probab. Rel. Topics 1 (1998) 663-670.

2. L. Accardi and Y. G. Lu, The Wigner semicircle law in quantum electro dynamics, Comm. Math. Phys. 180 (1996) 605-632, Volterra preprint, No. 126 (1992).

3. L. Accardi, Y. G. Lu and I. Volovich, The QED Hilbert module and interacting Fock spaces, IIAS Report No. 1997-008 (1997) International Institute for Advanced Studies, Kyoto.

4. L. Accardi and M. Nahni, Interacting Fock space and orthogonal polynomials in several variables, preprint (2002).

5. L. Accardi and M. Skeide, Interacting Fock space versus full Fock module, Volterra preprint, No. 328 (1998).

6. L. Gross, Abstract Wiener spaces, in Proc. 5th Berkeley Symp. Math. Stat. and Probab. 2, part 1 (Univ. of California Press, 1965), pp. 31-42.

7. S. Janson, Gaussian Hilbert Spaces, Cambridge Tracts in Math., Vol. 129 (Cambridge Univ. Press, 1997).

8. H.-H. Kuo, Gaussian Measures in Banach Spaces, Lecture Notes in Math., Vol. 463 (Springer-Verlag, 1975).

9. H.-H. Kuo, Integration in Banach spaces, in Notes in Banach Spaces, ed. H. Elton Lacey (University of Texas Press, 1980), pp. 1-38. 
10. I. E. Segal, Tensor algebras over Hilbert spaces, Trans. Amer. Math. Soc. 81 (1956) 106-134.

11. J. A. Shohat and J. D. Tamarkin, The Problem of Moments, Mathematical Surveys, Number I (Amer. Math. Soc., 1943).

12. T. J. Stieltjes, Recherches sur les fractiones continues, Ann. Fac. Sci. Toulouse 8 (1894), T-1-122 (1895) A5-47.

13. N. Wiener, The homogeneous chaos, Amer. J. Math. 60 (1938) 897-936. 